\title{
Submitted: High-resolution ultrasound of spigelian and groin hernias: Accepted: 28.11.2020 a closer look at fascial architecture and aponeurotic passageways
}

Published: 08.03.2021

\section{Keywords}

hernia, ultrasound, abdominal wall, groin

\author{
Riccardo Picasso ${ }^{1,2}$, Federico Pistoia ${ }^{1,2}$, Federico Zaottini ${ }^{1,2}$, \\ Sonia Airaldi ${ }^{3}$, Maribel Miguel Perez ${ }^{4}$, Michelle Pansecchi ${ }^{1,2}$, Luca Tovt ${ }^{1,2}$, \\ Sara Sanguinetti ${ }^{1,2}$, Ingrid Möller ${ }^{5}$, Alessandra Bruns ${ }^{6}$, Carlo Martinolii, ${ }^{1,2}$
}

${ }^{1}$ Department of Health Science (DISSAL), Università di Genova, Genova, Italy

${ }^{2}$ UO Radiologia - IRCCS Ospedale Policlinico San Martino, Genova, Italy

${ }^{3}$ Radiologia Ospedale San Paolo, Savona, Italy

${ }^{4}$ Unidad de Anatomía y Embriología Humana, Departamento de Patología y Terapéutica Experimental, Facultad de Medicina y Ciencias de la Salud (Campus de Bellvitge), Universitat de Barcelona, L'Hospitalet de Llobregat, Barcelona, Spain

${ }^{5}$ Instituto Poal de Reumatologia, Barcelona, Spain

${ }^{6}$ Division of Rheumatology, Sherbrooke University, Sherbrooke, Canada

Correspondence: Prof. Carlo Martinoli, MD, Department of Health Science-DISSAL, University of Genova, Viale Pastore 1. I-16132, Genova, Italy, UO Radiologia - IRCCS Ospedale Policlinico San Martino; tel.: +39010 555 8788, fax: +390105556620

DOI: 10.15557/JoU.2021.0008

\begin{abstract}
From the clinical point of view, a proper diagnosis of spigelian, inguinal and femoral hernias may be relevant for orienting the patient's management, as these conditions carry a different risk of complications and require specific approaches and treatments. Imaging may play a significant role in the diagnostic work-up of patients with suspected abdominal hernias, as the identification and categorization of these conditions is often unfeasible on clinical ground. Ultrasound imaging is particularly suited for this purpose, owing to its dynamic capabilities, high accuracy, low cost and wide availability. The main limitation of this technique consists of its intrinsic operator dependency, which tends to be higher in difficultto-scan areas such as the groin because of its intrinsic anatomic complexity. An in-depth knowledge of the anatomy of the lower abdominal wall is, therefore, an essential prerequisite to perform a targeted ultrasound examination and discriminate among different types of regional hernias. The aim of this review is to provide a detailed analysis of the fascial architecture and aponeurotic passageways of the abdominal wall through which spigelian, inguinal and femoral hernias extrude, by means of schematic drawings, ultrasound images and video clips. A reasoned landmark-based ultrasound scanning technique is described to allow a prompt and reliable identification of these pathologic conditions.
\end{abstract}

\section{Introduction}

Abdominal wall hernias represent a common issue in clinical practice, with a prevalence amongst the general population to be estimated at around $5 \%{ }^{(1,2)}$. The vast majority $(85 \%)$ of hernias occur in the groin, whereas only $15 \%$ of them are found in other areas of the anterior abdominal wall $^{(2)}$. Diagnostic imaging is increasingly used for investigating patients with suspected abdominal wall hernias, especially when atypical bumps or symptoms may lead the clinician to suspect other etiologies ${ }^{(3)}$. In this clinical context, ultrasound (US) has been regarded as a low-cost, rapid and very effective technique which is able to identify and categorize different types of herniations in exquisite 


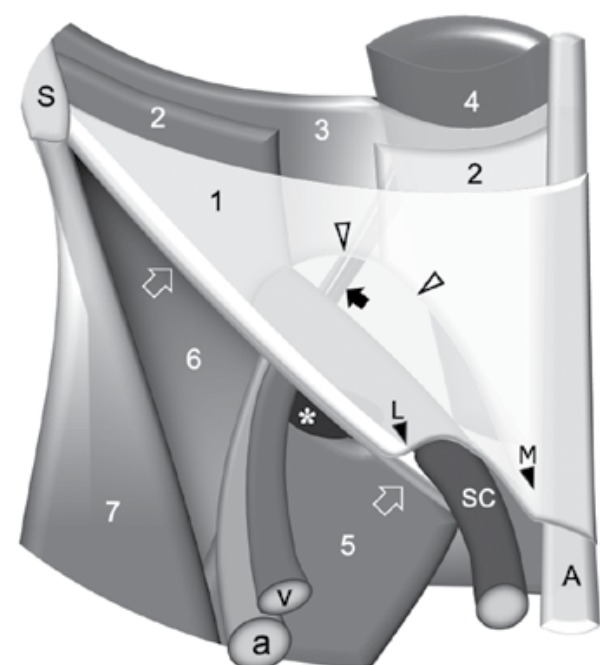

Fig. 1. Groin anatomy, anterior landmarks. Schematic drawing illustrates the respective arrangement and distal insertions of the anterior abdominal wall muscles (darker grays) and aponeuroses (lighter grays), including the obliquus externus (1) with its lateral $(L)$ and medial $(M)$ columns merging medially with the linea alba (A), the conjoint tendon (void arrowheads) of the obliquus internus (2) and the transversus abdominis (3). The aponeurosis of the obliquus internus is drawn discontinuous to allow cross visualization of the underlying transversus. Note the relationships of the aponeuroses with the rectus abdominis (4). The inguinal ligament (void arrows) gives insertion to the obliquus externus aponeurosis and separates the inguinal (up) from the crural (down) regions. After giving off the inferior epigastric vessels (black arrow), the common femoral artery (a) and vein (v) exit the pelvis crossing underneath the ligament. The femoral canal (asterisk) is a small opening roofed by the inguinal ligament that is reinforced medially by the Gimbernat's (lacunar) ligament (not shown). The pectineus muscle (5) forms the floor, and the femoral vein its lateral wall. In a more lateral position, the iliacus muscle (6) and the tensor fasciae latae (7) arising from the anterosuperior iliac spine (S) are shown. Medially, the spermatic cord (SC) is seen emerging from the external inguinal ring

anatomical detail ${ }^{(4-6)}$. The main advantage of US over computed tomography or MR imaging relies on the ability of this technique to provide a dynamic evaluation which may help to detect intermittent extrusions during the application of provocative maneuvers (i.e. Valsalva) and/or different patient positioning (i.e. supine vs. standing). The aim of this review is to place a specific focus on the complex fascial and aponeurotic anatomy of the anterior abdominal wall that is pertinent to spigelian, inguinal and femoral hernias. The anatomical background and the relevant US findings of the aponeurotic architecture and portals of herniation will be described using schematic drawings, static images and video clips.

\section{Anatomical considerations on the abdominal wall}

A deep knowledge of the anatomy of the anterior abdominal wall is mandatory to perform a targeted US examination

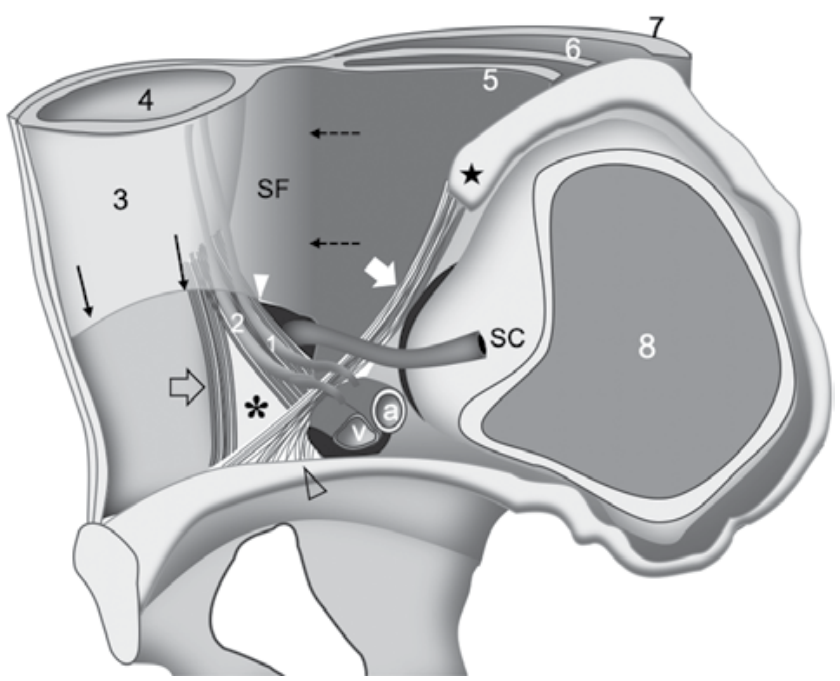

Fig. 2. Groin anatomy, posterior landmarks (modified from Netter, Atlas of Human Anatomy 2018). Schematic drawing illustrates the anterior abdominal wall from a posterior view. The internal inguinal ring (white arrowhead) is shown as an opening crossed by the spermatic cord (SC). More medially, the femoral artery (a) and vein (v) leave the pelvis through the lacuna vasorum, passing on the undersurface of the inguinal ligament (white large arrow) and lateral to Gimbernat's (lacunar) ligament (void arrowhead). Cranial to the inguinal ligament, the Hesselbach's triangle (asterisk) is delimited by the ligament of Henle (void arrow) medially, the Hesselbach's ligament (white arrowhead) and the inferior epigastric artery (1) and vein (2) laterally. After delimiting the side of the triangle, these vessels pierce the fascia of the rectus abdominis (4) at the level of the Douglas line (thin black arrows). The spigelian fascia $(S F)$ and the sharp and vertically oriented myotendinous junction (spigelian line) of the transversus abdominis (dotted black arrows) are indicated. Laterally, the bulk of the iliopsoas complex (6) leaves the pelvis through the lacuna musculorum. Note the multilayered arrangement of the myofascial planes of the anterior abdominal wall consisting, from depth to surface, of the posterior leaf of the obliquus internus and transversus abdominis aponeurosis (3), the transversus abdominis muscle (5), the obliquus internus (6) and the obliquus externus (7). Star, anterosuperior iliac spine

in patients with suspected ventral hernias. On each side of the linea alba, five paired muscles can be recognized in the abdominal wall: two of them - the rectus abdominis and the smaller pyramidalis - lie closer to the midline, with their major axis oriented in the craniocaudal direction. The other three muscles - obliquus externus, obliquus internus and transversus abdominis - are located more laterally: they are large, flattened, and more obliquely oriented. From the anatomical point of view, the right and left rectus abdominis muscles are separated in the midline by a vertical band of connective tissue which is referred to as the linea alba. They take their origin from the cartilages of the $5^{\text {th }}$ to $7^{\text {th }}$ ribs and the xyphoid process, and attach distally into the symphysis pubis, the pubic crest, and the pubic tubercle. The rectus abdominis is characterized by a series of transversely oriented connective tissue bands (tendinous intersections) that divide the muscle belly into parallel strips. In addition, it is enveloped and stabilized by the aponeuroses of the obliquus externus, obliquus internus, and transversus abdominis muscles. The pyramidalis is a small rudimentary 
muscle which lies distally, covering the anteroinferior aspect of the rectus. It has a triangular shape, arises from the linea alba, and attaches into the symphysis pubis and the pubic tubercle. The obliquus externus takes its origin from the lateral aspect of the last seven/eight ribs. It travels from cranial to caudal, and from lateral to medial, converging into a large and flattened aponeurosis that approaches the midline, crossing over the rectus abdominis and blending with the contralateral muscle at the level of the linea alba. The obliquus externus also has a distal insertion at the level of the groin. The obliquus internus muscle originates from the anterior and middle thirds of the iliac crest, the lateral half of the inguinal ligament and, through the thoracolumbar fascia, from the spinous processes of the last two lumbar and first sacral vertebrae. Its fibers diverge in a large fan and form a broad aponeurotic tendon that can be subdivided into proximal, intermediate and distal parts. The proximal part inserts into the costal cartilages of the last three/four ribs. In its superior two thirds, the intermediate part runs horizontally, and splits into posterior and anterior layers that envelop the rectus abdominis, whereas its inferior third simply crosses over this muscle, merging with the obliquus externus aponeurosis. The distal part of the obliquus internus aponeurosis inserts into the pubic crest and the pectineal line through a conjoint tendon with the transversus abdominis (Fig. 1). The transversus abdominis muscle is the innermost of the series of flat muscles of the anterior abdominal wall. It has an extensive origin from the internal aspect of the last six costal cartilages, the transverse processes of the lumbar vertebrae through the thoracolumbar fascia, the deep aspect of the anterior iliac crest, and the lateral half of the inguinal ligament. The transversus abdominis muscle travels from posterior to anterior to converge in a large aponeurosis that inserts into the linea alba. Its myotendinous junction has a medial concave shape that is commonly referred to as the spigelian or semilunaris line (Fig. 2). The area between the spigelian line and the lateral border of the rectus abdominis is called the spigelian fascia (Fig. 2). While the superior part of the aponeurosis runs on the undersurface of the rectus abdominis to reach the midline and insert into the linea alba, the inferior lies on the anterior aspect of the rectus. The arcuate line (Douglas line) indicates the transition between the intermediate and distal parts of the obliquus internus aponeurosis and the proximal and distal parts of the transversus abdominis aponeurosis. It demarcates the point at which the inferior epigastric vessels pierce the undersurface of the rectus (Fig. 2). The distal part of the transversus abdominis aponeurosis also shows a common insertion with the obliquus internus into the pubic crest and the pectineal line.

\section{Spigelian hernia}

The spigelian hernia derives from peritoneal infolding through a defect in the spigelian fascia ${ }^{(7-9)}$. This typically occurs along the Douglas line, as the change in orientation of the aponeuroses of the obliquus internus and transversus abdominis is supposed to cause focal weakening of the abdominal wall(10). The sac of spigelian hernias may contain the omentum, small bowel or colon ${ }^{(10)}$. Surgical repair is generally indicated due to a high risk of incarceration $(17-24 \% \text { of cases })^{(10)}$. Regarding presentation symptoms, patients often complain of pain at the level of the abdominal wall, and report an intermittently palpable mass. The clinical diagnosis is often challenging, as the protrusion may be hidden by the overlying normal aponeurosis of the obliquus externus. A low diagnostic accuracy of CT and US has also been reported due to the transitory presentation of this type of hernia that may or may not be present at the time of imaging $^{(11-15)}$. Placing the US transducer at the level of the inferior third of the rectus abdominis, the inferior epigastric vessels should be identified first, as they run vertically alongside the muscle undersurface. These vessels should then be followed on their short-axis from distal to proximal until they pierce the deep fascia of the rectus abdominis at the Douglas' arcade (Fig. 3). From this level, the probe should be moved externally on transverse planes, looking at the three-layered complex of aponeuroses of the obliquus externus, obliquus internus, and transversus abdominis. The myotendinous junction of the transversus abdominis indicates the level of the spigelian (semilunaris) line, whereas the area intervening between this line and the lateral margin of the rectus abdominis represents the spigelian fascia (Fig. 4). The spigelian hernia can be found at the level of the Douglas' arcade through a defect in the spigelian fascia (Fig. 5 and Video 1). If the suspected hernia is not visualized at this level, the spigelian fascia should be extensively examined during the Valsalva maneuver to avoid missing small or atypically located protrusions. Compression maneuvers are important to assess reducibility.

\section{Anatomical considerations on the inguinal area}

Several muscles, tendons and ligaments contribute to building up the complex three-dimensional architecture of the groin. A thorough anatomical knowledge is, therefore, a critical prerequisite to perform a correct evaluation and increase the examiner's confidence in diagnosing regional hernias. After inserting into the anterior third of the iliac crest and the anterior superior iliac spine, the obliquus externus aponeurosis gets thicker and directs inferomedially to reach the pubic tubercle, forming a strong tendinous arch between the two pelvic bones, which is referred to as the inguinal ligament (Fig. 1). Approaching the pubis, some fibers of the inguinal ligament reflect posteriorly to insert into the pectineal line. They form the lacunar ligament (Gimbernat's ligament) and delimit the medial wall of the femoral ring (Fig. 2). Close to its insertion into the pubic tubercle, the inguinal ligament also exhibits a thin extension (pectineal ligament or Cooper's ligament) which diverges toward lateral, and attaches onto the superior pubic ramus. This latter tendinous extension forms the floor of the femoral ring. In close proximity to the lateral side of the rectus abdominis, an opening in the distal obliquus externus aponeurosis contours the superficial inguinal ring a few millimeters cranial to the insertion of the inguinal ligament into the pubic tubercle. This opening is bounded by lateral, medial and posterior walls: the lateral wall consists of vertically-oriented fibers of the obliquus externus blending with the underlying 

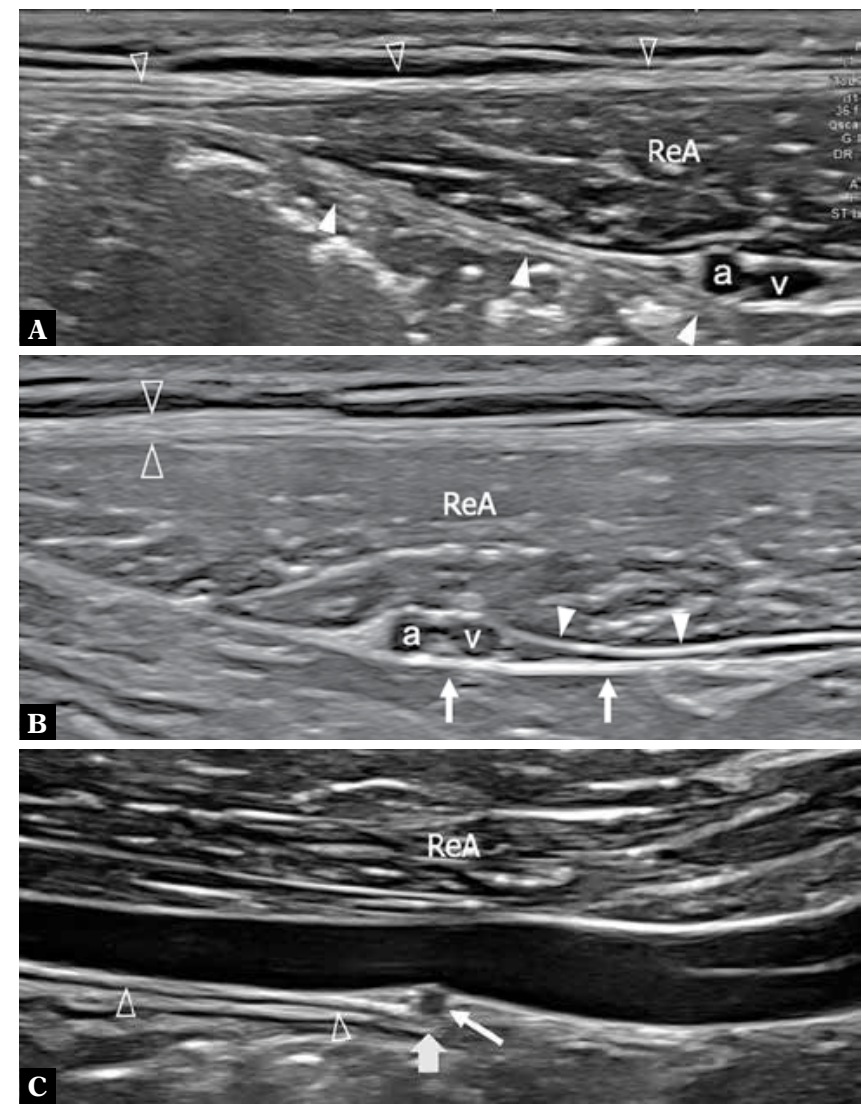

Fig. 3. Douglas line (arcuate line of rectus sheath or linea semicircularis). A, B. Transverse 18-5 MHz. US images obtained cranial (A) and caudal (B) to the Douglas line demonstrate the relationship of the inferior epigastric artery (a) and vein $(v)$ with the aponeurosis of the anterior abdominal wall muscles. A. the obliquus externus and the anterior leaf of the obliquus internus aponeurosis (void arrowheads) run toward the midline crossing over the muscle belly of the rectus abdominis (ReA), whereas the conjoint aponeurosis (white arrowheads) of the transversus and the posterior leaf of the obliquus internus reaches the linea alba passing on the undersurface of the rectus abdominis. B. the inferior epigastric artery (a) and vein (v) are seen running between the fascia transversalis (white arrowhead) and the parietal peritoneum (arrows), given that the aponeuroses of the abdominal wall muscles (void arrowheads) cross over the rectus abdominis (ReA). C. Magnified long-axis 18-5 MHz. US image of the inferior epigastric artery demonstrates its passage across the Douglas line (large arrow), with a small collateral branch (narrow arrow) arising at this level. Note the conjoint transversus and the deep leaf of the obliquus internus aponeurosis (arrowheads) running on the undersurface of the rectus abdominis cranial to the line

inguinal ligament, and the medial wall derives from more transversely-oriented fibers approaching the midline and inserting into the symphysis pubis (Fig. 1). Some of these latter fibers cross the midline and reach the pectineal line, forming the posterior wall (Colles' ligament) of the contralateral superficial inguinal ring. The distal aponeurosis of the obliquus internus and transversus abdominis share a common insertion into the pubis which is commonly referred to as the conjoint tendon or inguinal falx (Fig. 6). It lies superficial to the lateral boundary of the rectus abdominis, thus reinforcing the abdominal wall in the groin area. Finally, the
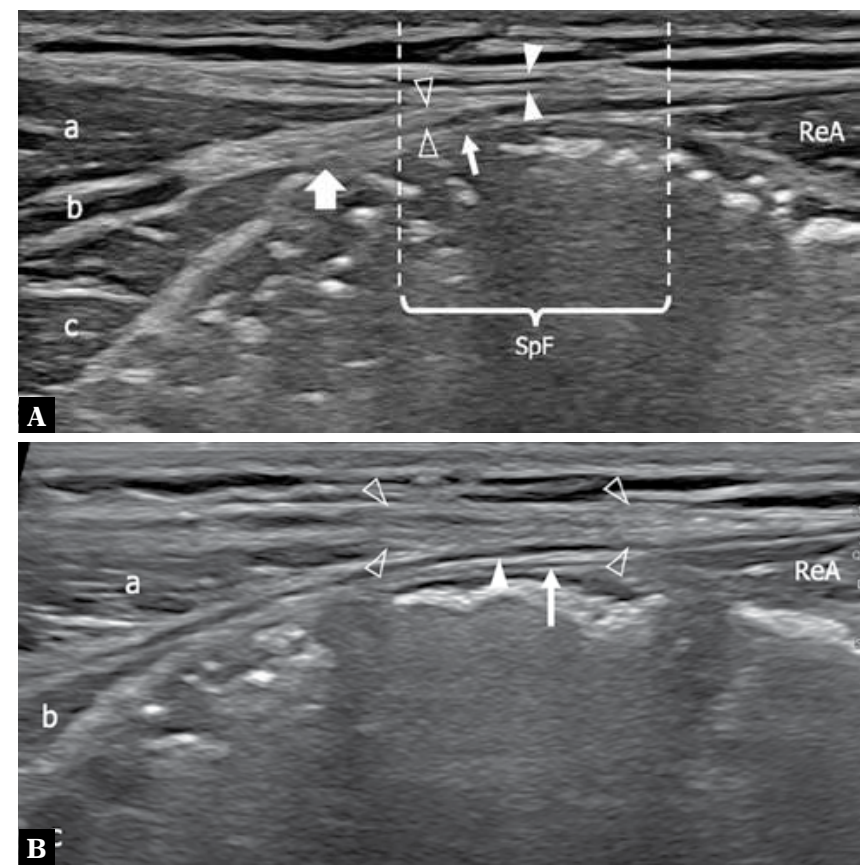

Fig. 4. Spigelian line and fascia. A. Transverse 18-5 MHz US image demonstrates the relationship of the obliquus externus ( $a$ ), obliquus internus (b), transversus abdominis (c) and their corresponding aponeuroses (white arrowheads, void arrowheads and thin white arrows respectively) with the rectus abdominis (ReA). The spigelian line (large white arrow) represents the level of the myotendinous junction of the transversus abdominis, whereas the spigelian fascia (SpF) consists of the transversus aponeurosis between the spigelian line and lateral border of the rectus abdominis (ReA). B. Transverse 22-8 MHz US image obtained distal to the Douglas line shows the flat aponeuroses of the obliquus externus (void arrowheads), obliquus internus (white arrowhead) and transversus abdominis (thin white arrow) as they cross over the rectus abdominis

deepest structure of the abdominal wall is the fascia transversalis, which lies on the posterior aspect of the transversus abdominis muscle. Looking at this fascia from the abdominal cavity, two thickened bands can be noted: the medial (Henle's ligament) is adjacent and parallel to the lateral margin of the rectus abdominis and corresponds, in a more superficial plane, to the inguinal falx; the lateral (Hesselbach's ligament) runs close and parallel to the inferior epigastric vessels (Fig. 2). These ligaments show an undefined proximal origin, and insert distally into the inguinal ligament, playing an important role as groin stabilizers. The internal inguinal ring can be considered an opening of the fascia transversalis: it is located on a plane crossing the midpoint of the inguinal ligament, approximately $11 \mathrm{~mm}$ above it, just lateral to the Hesselbach's ligament that contributes to its medial boundary. The inguinal canal travels through different layers of the abdominal wall, running from the deep (superolateral) to the superficial (inferomedial) inguinal rings. It houses the spermatic cord in men, as it travels from the abdomen to the scrotum, and the round ligament in women, directed to the labia majora. The canal has been described as having superior, inferior, anterior and posterior walls: the inferior wall is made of the inguinal ligament; the superior is composed of the inferior boundaries of the obliquus internus 
A

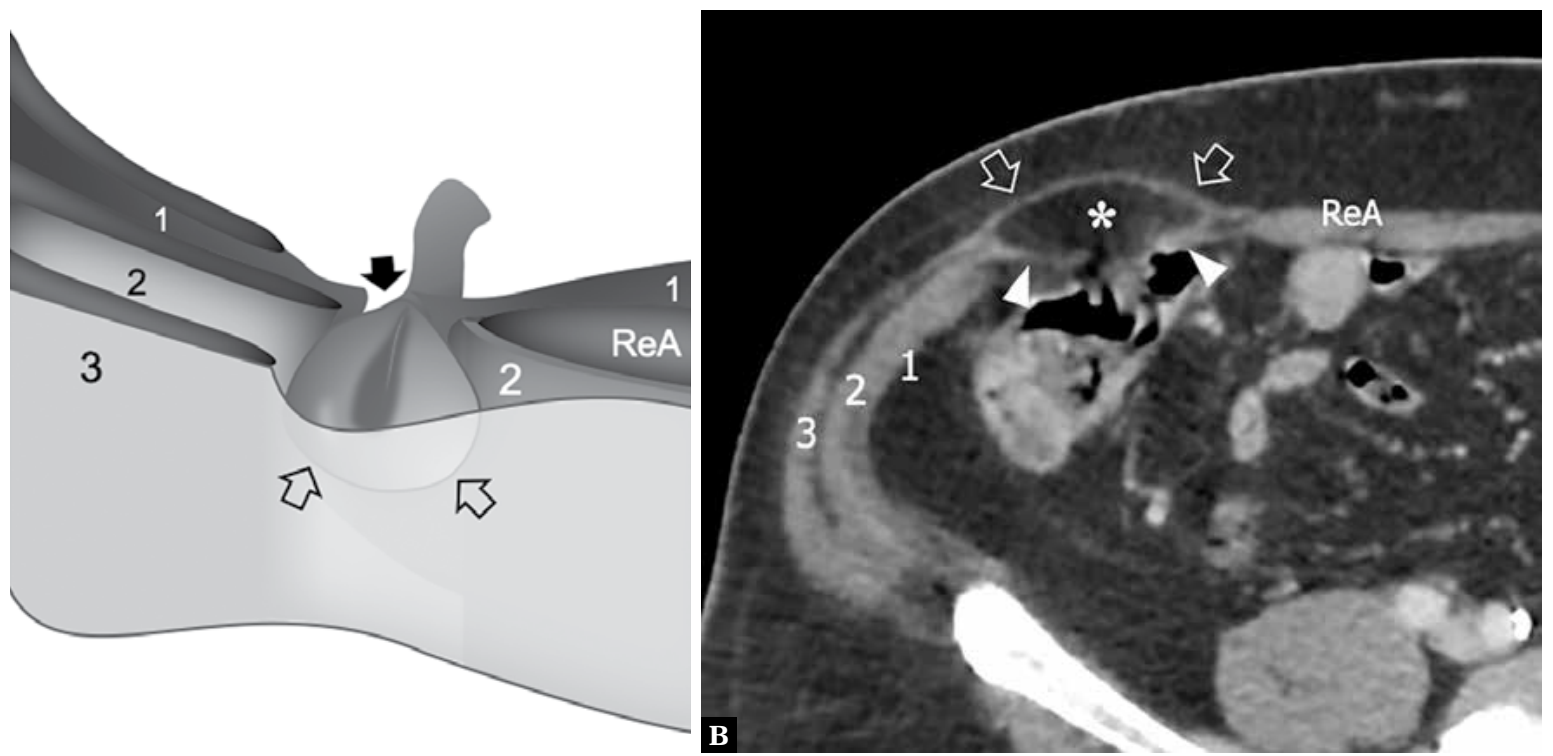

Fig. 5. Spigelian hernia. A. Schematic drawing illustrates a small bowel loop (void arrow) herniating through the spigelian fascia (black arrow). After crossing the transversus abdominis (1) and the obliquus internus (2), the hernia sac expands deep to the obliquus externus aponeurosis (3). ReA, rectus abdominis. B. Axial CT scan in a patient with recurrent episodes of abdominal pain and discomfort demonstrates a spigelian hernia (asterisk), protruding through a cleft in the spigelian fascia (arrowheads). The intact obliquus externus aponeurosis (void arrows) bounds the herniation, making it occult at physical examination. More laterally, the flat bellies of the transversus abdominis (1), obliquus internus (2), and obliquus externus (3) are seen

and transversus abdominis; the anterior is represented by the inferior part of the obliquus externus aponeurosis. The posterior wall has a more complex structure: i) its lateral third consists of the fascia transversalis reinforced by the Hesselbach's ligament and the inferior epigastric vessels; ii) its middle third is made up of the fascia transversalis, and iii) its medial third is composed of the fascia, the conjoint tendon, and Henle's ligament, which reinforce its superficial and deep aspects, respectively. Medial to the Hesselbach's ligament and the inferior epigastric vessels, the middle third of the posterior wall represents a weak area of the abdominal wall (Hesselbach's triangle), as the fascia transversalis lacks any reinforcement there (Fig. 2 and Fig. 6). This is the area through which direct hernias come out. Indirect inguinal hernia descends instead into the inguinal canal, passing through the deep inguinal ring. When dealing with a suspected groin hernia, it should be kept in mind that small protrusions may be unnoticed at rest, with the patient lying supine on the bench. To avoid false negatives, the groin area should also be systematically investigated during the Valsalva maneuver or when standing. US imaging demonstrates the inguinal ligament as an undefined thin hyperechoic band running from the anterior superior iliac spine to the pubic tubercle (Fig. 7). The external iliac vessels can be easily seen, as they cross underneath the middle third of the ligament. Just proximal to it, US can demonstrate the origin of the inferior epigastric vessels which turn cranially to run alongside the undersurface of the rectus abdominis. The inferior epigastric vessels represent the main landmark to identify the Hesselbach's triangle, which is located in the area intervening between them and the lateral margin of the rectus abdominis. Lateral and slight superior to the origin of the inferior epigastric vessels, the spermatic cord can be identified as an ill-defined hyperechoic structure housing vessels

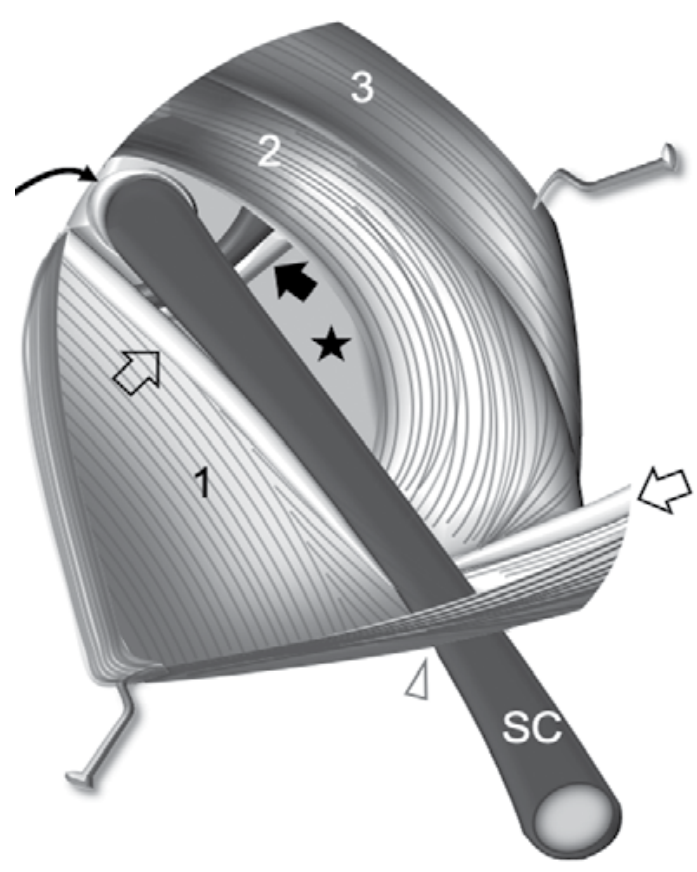

Fig. 6. Inguinal region and Hesselbach's triangle. Schematic drawing offers a closer look of the anatomical structures surrounding the spermatic cord (SC) in the inguinal canal. In this drawing, the aponeuroses of the obliquus externus (1) and the conjoint ones of the obliquus internus (2) and transversus abdominis (3) are artificially displaced by pins for better visualization of the field of interest. The spermatic cord (SC) is depicted between the internal (curved arrow) and external (arrowhead) inguinal rings, travelling across the aponeurotic layers and close to the inguinal ligament (void arrows). Note the Hesselbach's triangle (star), the base of which is lateral and formed by the inferior epigastric vessels (black arrow) 


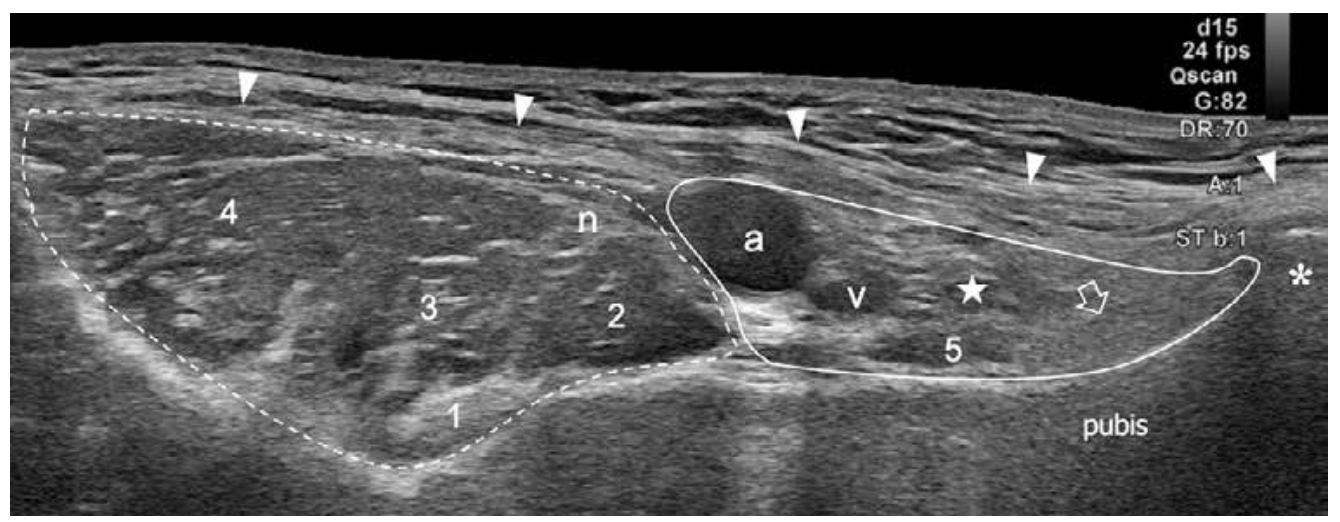

Fig. 7. Inguinal ligament. Oblique extended field-of view 18-5 MHz US image oriented between the anterosuperior iliac spine and the pubic tubercle (asterisk) demonstrates the inguinal ligament (white arrowheads) in its long-axis as it crosses the groin. The inguinal ligament bridges two wide passageways separated by the iliopectineal ligament (not shown). The lateral (lacuna musculorum) is crossed by the tendon (1) and most distal part of the myotendinous junction (2) of the psoas, the medial (3) and lateral (4) components of the iliacus, the femoral (n) and lateral femoral cutaneous nerves. The medial (lacuna vasorum) houses the femoral artery (a) and vein (v), the pectineus muscle (5), and the ligament of Cooper (void arrow) that is an extension of the inguinal ligament inserting into the pubic ramus and forming the floor of the femoral ring (star)

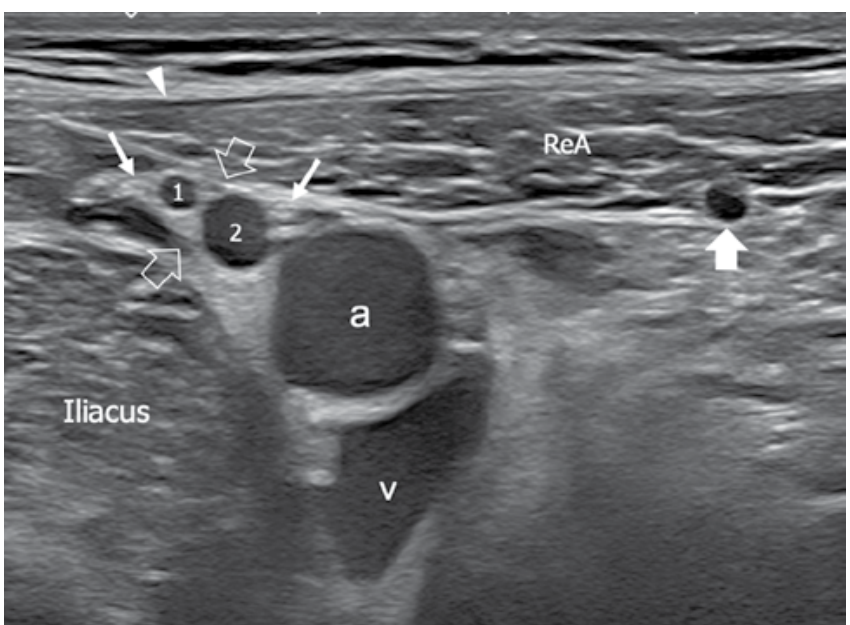

Fig. 8. Inguinal canal: internal inguinal ring level. Transverse 18-5 MHz US image demonstrates the spermatic cord large void arrows) emerging from the internal inguinal ring lateral to the external iliac artery (a) and vein (v). Several structures can be recognized inside the inguinal canal, including arteries (1), veins (2), and small superficial groin nerves (narrow arrows). Whereas the genital branch of the genitofemoral nerve travels from the internal to the external inguinal ring, thus crossing the whole inguinal canal, it is worth considering that the ilioinguinal nerve does not enter the canal through the internal ring and crosses only part of it. The inferior epigastric artery (white large arrow) runs in a more medial position relative to the internal inguinal ring, on the undersurface of the rectus abdominis (ReA)

(i.e. spermatic, deferential and cremasteric arteries, venous plexus) and thin hypoechoic non-vascular tubular structures related to the deferens and small nerves (i.e. genital branch of the genitofemoral, ilioinguinal running on its external surface). The inguinal canal begins at the deep inguinal ring and descends parallel to the inguinal ligament, pointing toward the pubic tubercle to end at the superficial inguinal ring, where the spermatic cord passes into the subcutaneous tissue (Fig. 8 and Fig. 9).
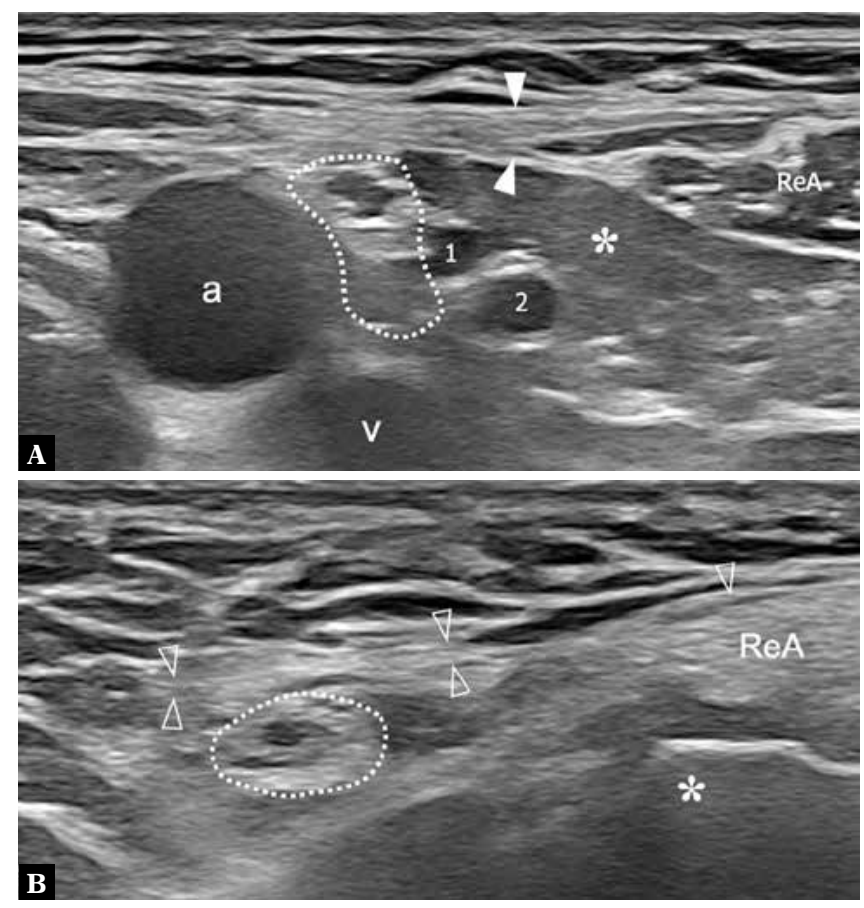

Fig. 9. Inguinal canal: middle third and external inguinal ring levels. A. Short-axis 18-5 MHz US scan shows the proximal third of the spermatic cord (dotted line) travelling inside the inguinal canal at the level of origin of the inferior epigastric vessels (arrows) from the external iliac artery (a) and vein (v). Hesselbach's triangle appears as a fat-filled area (asterisk) between the lateral border of the rectus abdominis (ReA) and the inferior epigastric vessels, where no structures other than the fascia transversalis restrain the abdominal content from protruding. The distal aponeurosis (white arrowheads) of the obliquus externus is shown bridging over the inguinal canal. B. Short-axis $18-5 \mathrm{MHz}$ US obtained at the level of the pubic tubercle (asterisk) demonstrates the spermatic cord (dotted line) approaching the external inguinal ring deep to the aponeurosis of the obliquus externus (void arrowheads). More medially, the insertion of the rectus abdominis (ReA) reinforced by fibers from the adductor longus and the contralateral obliquus externus appears as an echogenic band overlying the pubis 

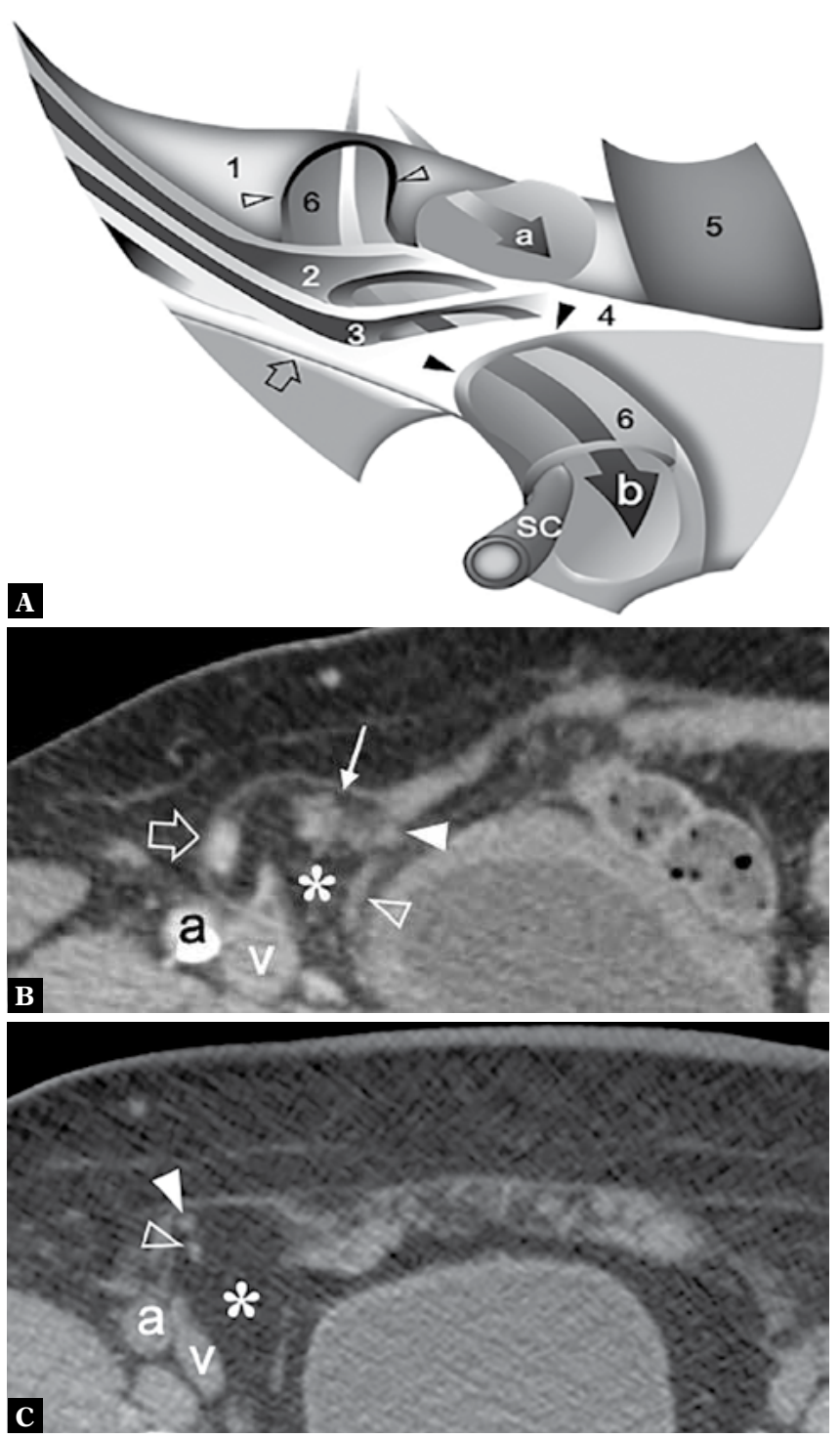

Fig. 10. Direct and indirect inguinal hernias. A. Schematic drawing of the groin illustrates the portals of direct (a) and indirect (b) inguinal hernias (modified from Netter, Atlas of Human Anatomy 2018). The indirect hernia runs across the whole inguinal canal (6), travelling from the internal (void arrowheads) to the external (black arrowheads) inguinal rings and displacing the spermatic cord (SC) on the side. The relationship of the inguinal canal with the series of local aponeurotic layers related to the fascia transversalis (1), transversus abdominis (2), obliquus internus (3) and obliquus externus (4) is illustrated. The direct hernia is seen forcing a passage through the fascia transversalis, in an area where this latter is not supported by any reinforcing structures, and expanding outside the inguinal canal, on the lateral side of the rectus abdominis (5). Void arrow, inguinal ligament. B, C. Oblique axial reformatted CT images from two different patients demonstrate the different pathways of direct (B) and indirect (C) inguinal hernias. B. The neck (asterisk) of the indirect hernia enters the canal external to the inferior epigastric artery (white arrowhead) and vein (void arrowhead). The spermatic cord (thin arrow) is displaced on the side of the canal. Arrow, groin lymph node. C. The neck (asterisk) of the direct hernia is seen expanding medial to the inferior epigastric artery (white arrowhead) and vein (void arrowhead). B, C. The relationship between the external iliac artery $(a)$ and vein $(v)$ and the hernia portals are also illustrated

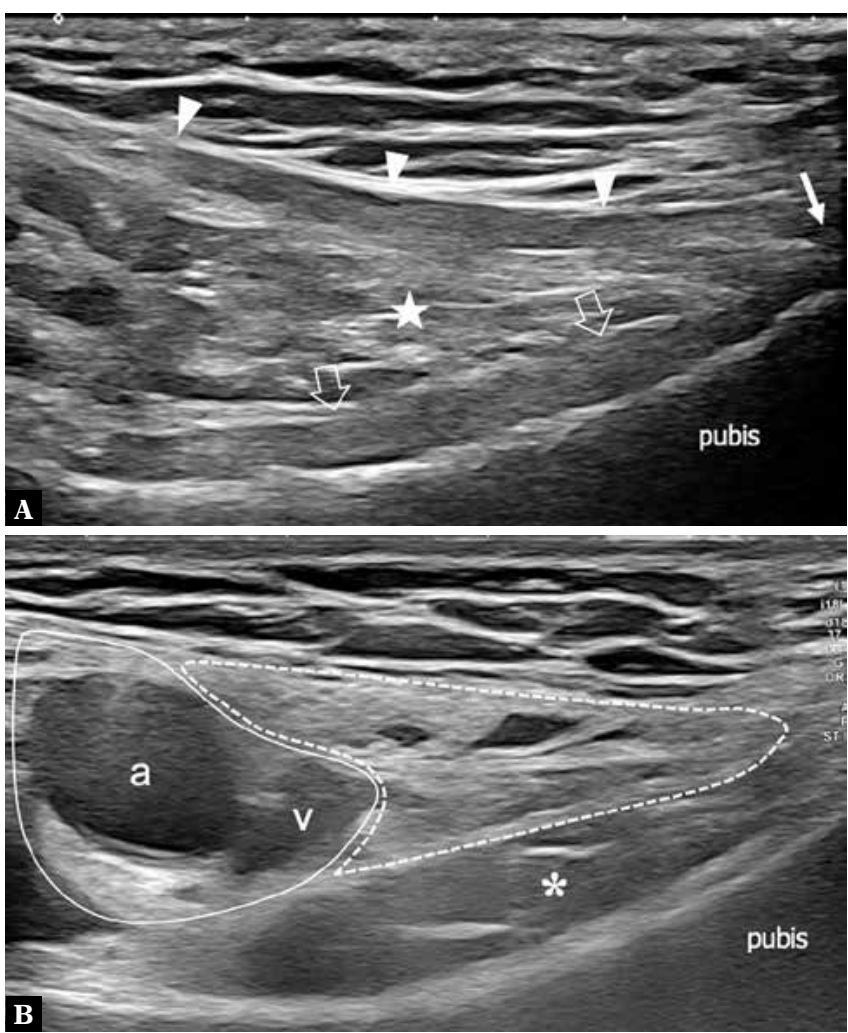

Fig. 11. Femoral ring and femoral canal. A, B. Transverse 18-5 MHz US images demonstrate the anatomical boundaries of the femoral ring (star) and canal (dotted line). The femoral ring forms the gate between abdomen and thigh. It is delimited by the Cooper's ligament (void arrows) posteriorly and the inguinal (white arrowheads) and Gimbernat's ligaments (thin white arrow) anteromedially. Soon after crossing the ring, the femoral canal lies anterior to the pectineus (asterisk) and medial to the femoral artery (a), vein (v) and branches of the femoral nerve which are invested by the femoral sheath to form a neurovascular compartment in continuity with the lacuna vasorum

\section{Indirect inguinal hernia}

Indirect inguinal hernias enter the inguinal canal through the deep inguinal ring, lateral to the position of the inferior epigastric vessels. In males, they traverse the canal and may extend beyond the external inguinal ring down to the scrotum (Fig. 10A and Fig. 10B). Indirect inguinal hernias are five times more common than direct hernias, and are reported to carry a moderate risk of strangulation ${ }^{(16)}$. The pathogenesis of indirect hernias differs between the children age group and the adult population. In children, herniation may be secondary to incomplete closure of the processus vaginalis, which is the part of the fascia transversalis that travels with the testes during their descent into the scrotum $^{(17,18)}$. Although a higher patency rate of the processus vaginalis is also found in adults ${ }^{(17,19)}$, a "shutter and sphincter" mechanism has been postulated in this latter age group to prevent the protrusion of the abdominal content when the intraabdominal pressure increases. This mechanism is mediated by the Hesselbach's ligament that is closely associated with the deep inguinal ring. When the transversus abdominis contracts, the ligament pulls the ring superolaterally, 

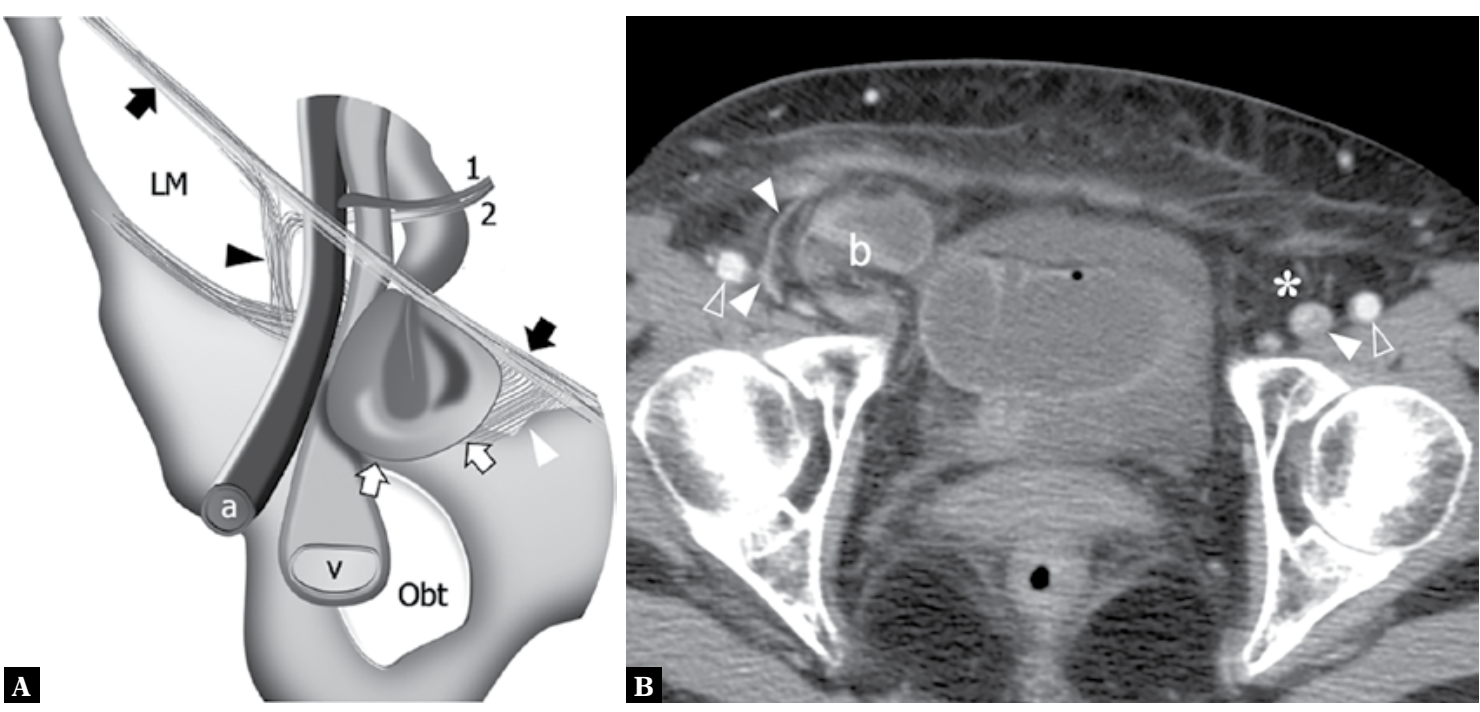

Fig. 12. Femoral hernia. A. Schematic drawing of the groin illustrates a small bowel loop (white arrows) crossing the femoral ring and expanding in the femoral canal, between the Gimbernat's ligament (white arrowheads), the inguinal ligament (black arrow), and the femoral vein (v). The femoral artery (a) runs more laterally in the lacuna vasorum, alongside the iliopectineal ligament (black arrowhead). In a more external position, note the lacuna musculorum (LM). 1, inferior epigastric artery; 2, inferior epigastric veins; obt, obturator foramen. B. Oblique axial reformatted CT in a patient who had acute intestinal obstruction demonstrates a distended bowel loops (b) herniating inside the femoral canal. The bulk of the hernia content compresses the adjacent femoral vein (white arrowheads), whereas the femoral artery (void arrowhead) remains unaffected. Asterisk, contralateral femoral canal

causing its narrowing and leading to an obstructed migration of the peritoneal content ${ }^{(20)}$. The relaxation of abdominal muscles with aging in conjunction with factors that increase the intraabdominal pressure and/or contribute to collagen degradation, such as coughing, straining, weightlifting and smoking, are implicated in decreasing the efficiency of this mechanism $^{(21,22)}$. US can categorize inguinal hernias as of indirect type when the sac is shown entering the inguinal canal through the deep inguinal ring (Video 2). The level of this ring can be recognized starting at the point where the inferior epigastric vessels arise from the medial aspect of the external iliac vessels. Sweeping the probe a few centimeters proximal to this level, the deep inguinal ring is easily found lateral to the external iliac artery and vein (Fig. 8). The probe should then be oriented obliquely along the inguinal canal to be in axis with the herniation path. Alternatively, one could scan the inguinal canal in its short-axis to identify the oval echogenic profile of the spermatic cord as a landmark. Moving proximally, the spermatic cord is seen crossing over the external iliac vessels and then pointing towards depth at the level of the deep inguinal ring. Indirect inguinal hernias include a wide range of content, such as retroperitoneal fat, omentum, bowel loops, appendix (Amyand's hernia), bladder, Meckel's diverticulum (Littré's hernia), gonads or the antimesenteric wall of a bowel loop (Richter's hernia) ${ }^{(16)}$.

\section{Direct inguinal hernia}

Direct inguinal hernias protrude through the Hesselbach's triangle, a weak area delimited laterally by the inferior epigastric vessels and the Hesselbach's ligament, medially by the lateral side of the rectus abdominis reinforced by the conjoint tendons (anteriorly) and the Henle's ligament (posteriorly), whereas its base consists of the inguinal ligament
(Fig. 10A and Fig. 10C). The Hesselbach's triangle can confidently be identified with US looking at the level of origin of the inferior epigastric vessels. On this scanning plane, the triangle is demonstrated as a fat-filled area extending between them and the rectus abdominis (Fig. 9A). The prevalence of direct hernias markedly increases with age as a result of degeneration and structural derangement of the fascia transversalis ${ }^{(23)}$. The pathogenesis of this condition seems to be related to the chronic continuing compression exerted by the abdominal content against the fascia transversalis, which shows chronic inflammatory infiltrates, microvascular damage, venous congestion, and impaired tissue perfusion ${ }^{(24-26)}$. These changes may lead to fascial weakening and breaking, with subsequent prominence of the abdominal content to impact against the deep aspect of the inguinal canal. Direct inguinal hernias most commonly occur in men, and have a low risk of strangulation, possibly because of their relatively large neck and the fact that they do not penetrate the inguinal canal ${ }^{(27)}$. US imaging visualizes the hernia as it expands lateral to the echogenic spermatic cord and medial to the landmark of the inferior epigastric vessels. Direct inguinal hernias typically appear as a bulge of fatty tissue or bowel causing "ballooning" of the posterior wall of the inguinal canal at the level of the Hesselbach's triangle (Video 3). The content of the inguinal canal may appear compressed and stretched laterally by the bulk of the hernia.

\section{Anatomical considerations on the crural (femoral) area}

Distal to the inguinal ligament, the fascia transversalis continues down into the thigh, where it is referred to as the "femoral sheath". It crosses over the femoral vessels 
for a few centimeters before fusing with their fascial envelopes. The "femoral canal" is delimited by the femoral sheath and located just medial to the femoral vein: it is bordered by the inguinal ligament, the pubic ramus covered by the Cooper's ligament proximally and the pectineus muscle distally, and the Gimbernat's (lacunar) ligament (Fig. 11). The femoral canal communicates with the abdominal cavity through the so called "femoral ring" that represents the passageway through which femoral hernias extrude (Fig. 11). Inferior to the inguinal ligament, several groups of lymph nodes can be visualized in the crural area. In normal states, these lymph nodes may appear oval or elongated in shape, and can be easily recognized as consisting of a peripheral hypoechoic rim (cortical layer) and a fatty hyperechoic center (hilum).

\section{Femoral hernia}

Femoral hernias are found below the inguinal ligament, and they protrude into the femoral canal through the femoral ring. Compared with inguinal hernias, femoral hernias are relatively uncommon, with a reported prevalence of approximately $3 \%$ of all groin hernias which need surgical repair ${ }^{(23)}$. Most occur in women as a probable result of their hormonal status and pregnancy-related changes which may lead to derangement and weakening of the supporting connective tissue around the femoral ring ${ }^{(28)}$. Although distinguishing inguinal from femoral hernias is not straightforward on clinical grounds regardless of the experience of the examining physician, a prompt diagnosis of the latter is crucial, as femoral hernias are associated with higher rates of acute complications ${ }^{(29)}$. US imaging has been reported to be highly accurate in differentiating between femoral and inguinal hernias if the examination is performed with an appropriate scanning technique and in experienced hands ${ }^{(30,31)}$. Since the risk for strangulation of femoral hernias accounts for $22 \%$ and $45 \%$ over three and twenty-one months respectively, whereas it occurs in

\section{References}

1. Everhart JE (ed.): Digestive diseases in the United States: epidemiology and impact. US Department of Health and Human Services, Public Health Service, National Institutes of Health, National Institute of Diabetes and Digestive and Kidney Diseases. Government Printing Office, Washington DC 1994.

2. Greenfield LJ: Review for surgery: scientific principles and practice. Lippincott Williams \& Wilkins, Philadelphia 2002.

3. Lassandro F, Iasiello F, Pizza NL, Valente T, Stefano ML, Grassi R et al.: Abdominal hernias: Radiological features. World J Gastrointest Endosc 2011; 3: 110-117.

4. Lee RK, Cho CC, Tong CS, Ng AW, Liu EK, Griffith JF: Ultrasound of the abdominal wall and groin. Can Assoc Radiol J 2013; 64: 295-305.

5. Jamadar DA, Jacobson JA, Morag Y, Girish G, Dong Q, Al-Hawarui M et al.: Characteristic locations of inguinal region and anterior abdominal wall hernias: sonographic appearances and identification of clinical pitfalls. AJR Am J Roentgenol 2007; 188: 1356-1364.

6. Young J, Gilbert AI, Graham MF: The use of ultrasound in the diagnosis of abdominal wall hernias. Hernia 2007; 11: 347-351.

7. Klinkosch JT: Divisionem herniarum novamgue herniae ventralis proponit. Dissertationum medicorum 1764: 184-186. only $3 \%$ and $4.5 \%$ of cases of inguinal hernias over the same time frame, it seems advisable to follow the policy of screening patients with suspected groin hernias systematically with $\mathrm{US}^{(32,33)}$. The examination should be performed by orienting the probe in the long-axis of the inguinal ligament, close to the pubic tubercle. Sweeping the probe slightly lateral and caudal to it, the common femoral artery and vein are visualized as they run underneath the ligament and superficial to the pubic ramus. The femoral ring can be recognized as the area bounded by the femoral vein laterally, the inguinal ligament anterosuperiorly and the Cooper's ligament posteriorly (Fig. 11A). When the bulk of a femoral hernia extrudes into the canal, the femoral vein collapses as a result of direct compression to the vessel walls (Fig. 12). During the Valsalva maneuver, the intermittent and reversible extrusion of the hernia with transient collapse of the vein can be nicely observed in dynamic scans (Video 4).

\section{Conclusion}

A clear differentiation amongst anterior abdominal wall and groin hernias is a critical issue, as these conditions often require different approaches and treatments. US imaging may be regarded as a useful complement to the physical examination for hernia characterization. Familiarity with the gross anatomy of the abdominal wall and the groin is a prerequisite to enhance the diagnostic confidence of the examiner when evaluating patients with suspected hernias.

\section{Conflict of interest}

Authors do not report any financial or personal connections with other persons or organizations, which might negatively affect the contents of this publication and/or claim authorship rights to this publication.
8. Gillispie CC: Dictionary of scientific biography, Volume XII. Charles Scribner's Sons, New York 1975.

9. Spangen L: Spigelian hernia. World J Surg 1989; 13: 573-580.

10. Larson DW, Farley DR: Spigelian hernias: repair and outcome for 81 patients. World J Surg 2002; 26: 1277-1281.

11. Balthazar EJ, Subramanyam BR, Megibow A: Spigelian hernia: CT and ultrasonography diagnosis. Gastrointest Radiol 1984; 9: 81-84.

12. Luedke M, Scholz FJ, Larsen CR: Computed tomographic evaluation of Spigelian hernia. Comput Med Imaging Graph 1988; 12: 123-129.

13. Shenouda NF, Hyams BB, Rosenbloom MB: Evaluation of Spigelian hernia by CT. J Comput Assist Tomogr 1990; 14: 777-778.

14. Torzilli G, Carmana G, Lumachi V, Gnocchi P, Olivari N: The usefulness of ultrasonography in the diagnosis of the Spigelian hernia. Int Surg 1995; 80: 280-282.

15. Losanoff J, Kjossev K, Handijev S, Karamfilova R: The diagnosis of Spigelian hernia $(\mathrm{SH})$ by high-resolution real-time sonography. J Ultrasound Med 1998; 17: 599-600.

16. Burkhardt JH, Arshanskiy Y, Munson JL, Scholz FJ: Diagnosis of inguinal region hernias with axial CT: the lateral crescent sign and other key findings. Radiographics 2011; 31: E1-12. 
17. van Wessem KJ, Simons MP, Plaisier PW, Lange JF: The etiology of indirect inguinal hernias: congenital and/or acquired? Hernia 2003; 7: 76-79.

18. Brendan Devlin H, Kingsnorth A, O'Dwyer PJ, Bloor K: Epidemiology and aetiology of primary groin hernias in adults. In: Management of Abdominal Hernias, 2nd ed. Chapman \& Hall Medical, London 1998.

19. Abrahamson J: Etiology and pathophysiology of primary and recurrent groin hernia formation. Surg Clin N Am 1998; 78: 953-972.

20. Spangen L: Shutter mechanisms in the inguinal canal. In: Arregui ME, Nagan RF (eds): Inguinal Hernia: Advances or Controversies. Radcliffe Med Press, Oxford 1995: 55-59.

21. Klinge U, Zheng H, Si ZY, Bhardwaj R, Muys L, Klosterhalfen B: Altered collagen synthesis in fascia transversalis of patients with inguinal hernia. Hernia 1999; 3: 181-187.

22. Read RC: The metabolic role in the attenuation of transversalis fascia found in patients with groin herniation. Hernia 1998; 2 (Suppl 1): 17.

23. Burcharth J, Pedersen M, Bisgaard T, Pedersen C, Rosenberg J: Nationwide prevalence of groin hernia repair. PLoS One 2013; 8: e54367.

24. Amato G, Agrusa A, Romano G, Salamone G, Cocorullo G, Mularo SA et al.: Histological findings in direct inguinal hernia. Hernia 2013; 17: 757- 763.

25. Amato G, Agrusa A, Romano G, Salamone G, Gulotta G, Silvestri F et al.: Muscle degeneration in inguinal hernia specimens. Hernia 2012; 16: 327-331.
26. Amato G, Romano G, Salamone G, Agrusa A, Saladino VA, Silvestri F et al.: Damage to the vascular structures in inguinal hernia specimens. Hernia 2012; 16: 63-67.

27. Wechsler RJ, Kurtz AB, Needleman L, Dick BW, Feld RI, Hilpert PL et al.: Cross-sectional imaging of abdominal wall hernias. AJR Am J Roentgenol 1989; 153: 517-521.

28. Zarvan NP, Lee FT, Yandow DR, Unger JS: Abdominal hernias: CT findings. AJR Am J Roentgenol 1995; 164: 1391-1395.

29. Hair A, Paterson C, O'Dwyer PJ: Diagnosis of a femoral hernia in the elective setting. J R Coll Surg Edinb 2001; 46: 117-118.

30. Bradley M, Morgan D, Pentlow B, Roe A: The groin hernia - an ultrasound diagnosis? Ann R Coll Surg Engl 2003; 85: 178-180.

31. Djuric-Stefanovic A, Saranovic D, Ivanovic A, Masulovic D, Zuvela M, Bjelovic M et al.: The accuracy of ultrasonography in classification of groin hernias according to the criteria of the unified classification system. Hernia 2008; 12: 395-400.

32. Gallegos NC, Dawson J, Jarvis M, Hobsley M: Risk of strangulation in groin hernias. Br J Surg 1991; 78: 1171-1173.

33. Whalen HR, Kidd GA, O'Dwyer P: Femoral hernias. BMJ 2011; 343: 1271-1273. 